solution is assisted by the presence of the moths Zygaena purpuralis and Miana expolita in the west of Ireland, and in Rhum. Moreover, the distribution of the two bees Bombus smithianus and $B$. jonellus var. hebridensis hints that the Shetland Islands cannot be neglected in the same discussion.

In our evolutionary studies, we have been struck by the unusual facies presented by many island species of animals and plants when they are studied alongside representatives of ordinary English populations, and we have already described well-marked island races, for example, an Outer Island honeysuckle Lonicera Periclymenum var. Clarki, a new race of Nyssia zonaria and so on, the whole emphasizing the importance both of the local race, and of isolation, in evolution. From the very nature of this work, observation must be backed by experimental work, and this, in the case of some forms, is well in hand.

\title{
Photochemical Reactions in the Gaseous, Liquid and Solid States*
}

\section{By Dr. C. F. Goodeve, University College, London}

DHOTOCHEMISTRY is concerned with processes in which light quanta are absorbed and chemical changes result. A molecular species is characterized by a certain spatial arrangement of atoms governed by forces due to electrons. The oscillating electric field of a light quantum acts on a binding electron and raises it to an energy-level where the bond is either loosened or broken. This permits the spatial rearrangements to take place which we observe as chemical change.

The photochemical process may be divided into two stages: the primary process which occurs more or less simultaneously with the absorption process, and the secondary process which occurs consequent to the first. These two processes can be studied separately and by different methods, the first by a study of the absorption spectrum and the second by the methods of reaction kinetics.

\section{Photosensitivity}

The dual nature of photochemistry is emphasized by a consideration of the velocity of photochemical reactions. This velocity generally follows the equation

$$
-\frac{d n}{d t}=\gamma I_{a}
$$

where $n$ represents the number of molecules of absorbing species present at any moment, $-\frac{d n}{d t}$ their rate of decrease with time, and $I_{a}$ the absorbed light intensity in quanta per second. $\gamma$ is the quantum efficiency defined as

$$
\frac{\text { No. of molecules changed }}{\text { No. of quanta absorbed }} \text {. }
$$

* Taken from a course of afternoon lectures given at the Royal Institution, February 16, 23, March 2. References to, and further details of, most of the work described here may be found in papers chemistry ( $J$. Phys. Chem. June 1938), and recent papers by Bowen, by Norrish and by the author, each with collaborators.
If a beam of monochromatic light of intensity $I$ quanta per second falls on a thin layer of absorbing substance the concentration of which is $n / v$ molecules per c.c., the fraction of the light absorbed is proportional to the concentration and to the thickness, and is given by

$$
\frac{I_{a}}{I}=\alpha \cdot \frac{n}{v} \cdot l
$$

The constant of proportionality $\alpha$ is the molecular extinction coefficient and has a value usually between $10^{-16}$ and $10^{-22} \mathrm{~cm} .{ }^{2}$. It is the 'target area' that a molecule presents to the light beam, that is, the chance of it absorbing light.

We can combine equations (1) and (2) to give us the final equation for the rate of a photochemical change

$$
-\frac{d n}{n d t}=\alpha \gamma \frac{I l}{v}=\alpha \gamma \cdot \frac{I}{A},
$$

where $I / A$ is the intensity per unit area. This is the simplest equation for the rate of a photochemical reaction, and it is to be noted that the rate-determining constant is the product $\alpha \gamma$. This combined quantity is obviously the photosensitivity.

We see therefore that the sensitivity of a substance to light is governed by two quantities : the chance of the substance absorbing a light quantum, and the efficiency of the process per quantum absorbed. For example, the well-known reaction which takes place when a mixture of chlorine and hydrogen is illuminated is a very fast one. The extinction coefficient of chlorine is comparatively low-about $10^{-21} \mathrm{~cm} .{ }^{2}$. This low extinction is, however, counterbalanced by the fact that the quantum efficiency in the reaction with hydrogen is very high, between $10^{3}$ and $10^{5}$. The photosensitivity therefore has values from $10^{-18}$ to $10^{-16} \mathrm{~cm} .^{2}$ per quantum absorbed. It follows from equation (3) that in bright daylight, the blue light 
of which has a value of $I / A$ of about $10^{15}$ quanta per sec. per $\mathrm{cm} .{ }^{2}$, chlorine reacts at a rate of about 10 per cent per second.

As another example, a very light-sensitive substance called visual purple can be extracted from the retinæ of the eyes of animals which have been adapted to darkness before being killed. It has recently been shown that the high sensitivity of this substance arises from the fact that the extinction coefficient is high, while the quantum efficiency has a value of about unity; its photosensitivity is about $10^{-16} \mathrm{~cm} .^{2}$. On the other hand, for reactions such as the photodecomposition of aliphatic nitroso compounds, the quantum efficiency is unity and the extinction coefficient very low, the value of the photosensitivity being less than $10^{-19} \mathrm{~cm} .^{2}$. If a solution of a nitroso compound is exposed side by side with a solution of visual purple, it decomposes at a rate 1,000 times slower than the visual purple, although its quantum efficiency is about the same. In a further class, there exist many dyestuffs of a very high extinction but of a low photosensitivity arising from the fact that their quantum efficiencies are extremely small. The photosensitivity of a reaction varies greatly with wave-length, largely due to the variation in the extinction coefficient $\alpha$.

Here, as in thermal reactions, it is more convenient to make observations on the number of molecules present after certain intervals of time rather than to measure the rate at any instant, and we therefore use an integrated form of equation (3). For a layer of any thickness we arrive at the equation,

$$
\log _{10} \frac{I_{t}}{I-I_{t}}=\alpha \gamma \cdot \frac{I}{2 \cdot 3 \mathrm{~A}} \cdot t+\text { constant, }
$$

where $I_{t}$ is the light transmitted by a substance at the time $t$. In practice, coloured products may be produced, impurities may be present, reactions independent of light may proceed, etc. Equations may be set up for all these types of photochemical change and in them the photosensitivity $\alpha \gamma$ is the major rate-determining constant, similar to the 'velocity constant' appearing in equations for firstand second-order reactions. The photosensitivity may be conveniently determined by plotting the logarithmic function against the time, when a straight line should be obtained the slope of which is equal to the photosensitivity times the light intensity, $I / A$. This analytical method has been called "the method of photometric curves"

\section{The Primary Process}

The absorption spectra of molecules in the gaseous state are of two principal types-discontinuous (band) and continuous. The first type can be further divided according as the bands can or cannot be resolved into lines by high dispersion instruments. The presence of bands indicates that the molecule on absorption of a quantum has vibration frequencies characteristic of the excited state, and indeed these frequencies can be determined from the intervals between the bands. The excited state must therefore be one that can vibrate-it must be a complete molecule although the valence bonds may be weakened.

Cases where the bands cannot be resolved have been interpreted as meaning that the molecule has a short life in the excited state-something of the order of $10^{-12}$ sec. This has been called predissociation. Conclusions in these cases, however, are not so reliable as in the others, as lack of resolution may arise from a number of causes.

If, on the other hand, there are no bands and the spectrum is continuous, there can be no characteristic frequency for the excited state and at least one of the bonds is broken. The primary process in this case yields atoms or free radicals.

\section{Secondary Processes}

Secondary processes following discontinuous absorption usually consist of one or other of the following :

(a) The molecule may fluoresce, that is, give out most of its excess energy as light and return more or less to its original state.

(b) The molecule may suffer a collision in which its excess energy is converted into thermal energy. It is thus deactivated and cannot fluoresce or undergo photochemical change. Usually a molecule of the same species is particularly effective in quenching fluorescence. This arises from the fact that it is attracted to an excited molecule.

(c) The collision may lead to a chemical change. In any system there is usually competition between these possibilities. Most molecules will fluoresce unless they are deactivated or undergo chemical change within $10^{-8}$ sec.

Considerable advance has recently been made in our knowledge of the mechanism of chemical changes occurring on collision. This advance has been based on a consideration of the potential energy of the system as a function of the distances between the atoms. For each chemical state, defined by the existence of chemical bonds between certain atoms, there is a 'multi-dimensional potential energy surface' which describes this functional relation. A change over from one state to the other only occurs at an intersection of these surfaces, as it is only here that the energy, position and momentum of each of the particles can best be conserved. We now know that these quantities need only be conserved within the limits 
set by the 'uncertainty principle' of quantum mechanics, and that change can take place with decreasing probability a short distance on either side of the intersection. In addition, however, the spins of the electrons need to be conserved.

Secondary processes following continuous $a b$ sorption can also be considered according to the methods of reaction kinetics. The atoms or free radicals, produced by the primary process, are very reactive as they have free valencies. The chance of their reacting depends on the chance of their suffering a collision with a species with which they could react, and on the chance of their having sufficient energy, $E_{c}$, to overcome any repelling force that may be present. This latter chance is given by the simple exponential expression, $\mathrm{e}^{-E_{\varepsilon} / \boldsymbol{R} T \text {. }}$

\section{EXAMPLES IN GASES}

Iodine and oxygen absorb discontinuously in certain parts of the spectrum; iodine has been found to fluoresce at very low pressures. The excited molecules formed by the primary (light absorption) process collide and react with normal molecules, forming $\mathrm{I}_{3}+\mathrm{I}\left({ }^{2} P_{3 / 2}\right)$ and $\mathrm{O}_{3}+\mathrm{O}\left({ }^{1} D\right)$ respectively in conformity with the conservation rules of the previous section. $I_{3}$ is, however, unstable, but ozone is produced with an efficiency of nearly two molecules per quantum absorbedthe second coming from a reaction between the oxygen atom and an oxygen molecule.

The bands of formaldehyde are unresolvable below $280 \mathrm{~m} \mu$. It is easily shown that the potential energy surface of the excited molecule intersects that of a different state of chemical combination of the four atoms $-\mathrm{H}_{2}+\mathrm{CO}$. A path therefore exists via which the formaldehyde molecule, on absorption of a light quantum, can change over spontaneously into hydrogen and carbon monoxide with good conservation of energy, position, momentum and electron spin, and indeed experimentally this change is found to occur with a quantum efficiency of unity.

Hydrogen iodide absorbs continually and therefore dissociates into two atoms. Energy considerations indicate that the hydrogen atom reacts on its first collision with an HI molecule to produce $\mathrm{H}_{2}+\mathrm{I}$. On the other hand, a reaction between $\mathrm{I}+\mathrm{HI}$ would be endothermic by $35 \mathrm{kcal}$. The value of $E_{c}$ is therefore at least as great as this and of $e^{-E_{c} / \boldsymbol{R} T}$ less than $10^{-26}$. This reaction is therefore negligible and the iodine atoms accumulate until they can collide and combine together. A quantum efficiency of two molecules of $\mathrm{HI}$ decomposed per quantum absorbed is therefore to be expected and is found experimentally over a wide range of pressure and temperature.
Although the chlorine-hydrogen reaction has been known for nearly a hundred years, it has only recently been understood. Most of the quanta responsible for photochemical change are absorbed in the continuous part of the spectrum and therefore the primary process is the production of chlorine atoms. Each atom suffers frequent collisions with hydrogen molecules, and from independent experiments it is found that about one collision in 10,000 is effective in producing a hydrogen chloride molecule and a hydrogen atom. The hydrogen atom reacts readily with a chlorine molecule, producing hydrogen chloride and a chlorine atom, which in turn repeats the cycle. In other words, a 'chain' is set up which can carry on indefinitely unless something happens to stop it. Most of the disagreeing results obtained by different investigators are now attributed to varying effects of chain-stoppers such as oxygen molecules or the walls of the container. With poisoned walls, and gases free from inhibitors, quantum efficiencies of $10^{\circ}$ have been obtained.

\section{REACTIONS IN LiQuidS}

In passing now to examples in the liquid state, we have first to consider how far our conclusions with regard to the primary process, as judged from absorption spectra, can apply. Molecules in the liquid are in a continual state of collision. As a consequence of this there is a general smoothing out of the absorption spectrum, and it is impossible to resolve the bands into fine lines, as in the case of discontinuous absorption in gases. However, a continuous absorption band of a molecule in the gaseous phase is characterized by the fact that it has a smooth rounded maximum and that its threshold on the long-wave side has a gentle slope. These two characteristics are retained in the liquid state, and in fact whenever we find, in liquids, absorption bands with rounded tops and gentle sloping thresholds, we find that photodecomposition takes place. For example, the absorption spectra of nitroso-compounds and diazonium salts are of this type. On the other hand, the spectra of most dyes, such as the cyanine dyes, have sharp maxima and steep thresholds. The primary process undoubtedly leaves the molecule intact and in an excited state. In the case of most dyes we find that the secondary process is one of fluorescence or deactivation, but sometimes the substances react with inorganic ions. The mechanism of the nitroso-compounds is not fully understood, but apparently free radicals are produced which react with the solvent.

A large amount of work has recently been done on the photodecomposition of aldehydes and ketones. Gaseous acetone decomposes with a 
quantum efficiency of about $0 \cdot 3$, and the reaction apparently proceeds by the following mechanism :

$$
\begin{aligned}
\mathrm{CH}_{3} \mathrm{COCH}_{3}+h \nu & \rightarrow \mathrm{CH}_{3} \mathrm{CO}+\mathrm{CH}_{3}, \\
\mathrm{CH}_{3} \mathrm{CO} & \rightarrow \mathrm{CH}_{3}+\mathrm{CO}, \\
2 \mathrm{CH}_{3} & \rightarrow \mathrm{C}_{2} \mathrm{H}_{6} .
\end{aligned}
$$

Pure liquid acetone, however, is completely inactive to light. Apparently on absorption of light the electronic energy is degraded to heat very quickly. We have already noted that molecules of the same species have a very powerful effect in deactivating one another.

Even in dilute solutions, however, this reaction, particularly with higher ketones, is largely suppressed. This is apparently due to the fact that the free radicals produced in the primary process, being caged around by solvent, very readily recombine with one another, thus reducing the quantum efficiency. This is a common type of phenomenon in photochemistry, and is due simply to the fact that the movement of molecules, or their parts, is restricted by the surrounding molecules. We could not get a permanent chemical change unless the parts of the molecules could stay apart long enough to enter into some other state of combination. Given sufficient thermal energy, however, they may react at once with caging solvent molecules.

On the other hand, the higher ketones decompose partially in another way.

$$
\sum_{\mathrm{CH}_{3}}^{\mathrm{CH}_{3} \mathrm{CH}_{2} \mathrm{CH}_{2} \mathrm{CH}_{2}} \rightarrow \stackrel{\mathrm{CH}_{3} \mathrm{CH}: \mathrm{CH}_{2}}{\mathrm{CH}_{2}}+\underset{\mathrm{CH}_{3}}{\mathrm{CH}_{3}}
$$

The process is a curious one. Light absorption takes place without a doubt by an electron associated with the carbonyl group. This excitation energy by some means or other is able to move along the molecule and break it at a position several carbon atoms away (a similar process occurs in crystals). The products are not free radicals, but normal molecules, and even if they are caged around by other molecules, they should have no tendency to recombine. We should not expect the rate of this process to be different in solution from what it is in the gaseous phase, and in fact no difference is found.

Finally, there is an interesting class of photoreactions in solution involving changes of the charge on an ion. On the face of it, these reactions should not involve movement of nuclei. For example, to change an iodide ion into an iodine atom requires only the removal of an electron. The same is true for the conversion of ferrous into ferric ion. These changes of charge, however, are accompanied by changes in the arrangement of the solvent sheath around the ions.

\section{Reactions in Solids}

So far we have seen that modern theories of photochemistry give a fairly satisfactory treatment of gaseous reactions, and, to some extent, of reactions in liquids. With solids, however, the theories are only in a very early stage of development. The chief difficulty is that, whereas in gases and in liquids we can deduce something about the primary process from the absorption spectra, parallel deductions with solids can only be made in exceptional cases. We know, of course, that the movement of an electron is involved, but we cannot deduce how far an electron acts alone and how far its movement is coupled with that of the nuclei. However, we should expect to find more deactivation and greater restriction to spatial rearrangement in solids. For example, trans. azobenzene on absorption of a light quantum changes over quantitatively into cis-azobenzene, and the latter on absorption is reconverted back into the trans form. Both these reactions involve a large spatial rearrangement of nuclei, a rearrangement which could not take place inside a crystal lattice. In fact, in the solid state, these substances are quite stable to light, the energy of the light being degraded into heat. Similarly, the quantum efficiency of the decomposition of ethyl iodide is reduced in the solid state.

On the other hand, we have reactions of the type such as the conversion of $o$-nitrobenzaldehyde into $o$-nitrosobenzoic acid. This reaction proceeds in solution with a quantum efficiency of about unity. It involves only an internal rearrangement of the position of an oxygen atom, a movement in no way obstructed in the crystalline state The conversion is found to proceed in the solid at exactly the same rate as in solution. There are many phototropic effects in organic chemistry which are of this type.

Apparently the only well-established case where atoms may be rearranged inside an ionic crystal is that of the dissociation of potassium hydride dissolved in potassium bromide. Here hydrogen and potassium atoms are produced by the action of light; these at low temperatures simply recombine. At room or higher temperatures, however, the hydrogen atoms have sufficient thermal energy to escape from their lattice points, the remaining potassium atoms producing a blue colour in the crystal.

While spatial rearrangements are restricted inside a crystal, they are much less so on the surface. However, the quanta absorbed by the surface layer of a crystal are generally few compared with the total number absorbed by the crystal itself, and could not account for most of the photochemical reactions occurring with solids. It is therefore believed that a quantum may be absorbed 
in the interior of a crystal and its energy passed on, more or less as a complete unit, to the surface.

There are two possible mechanisms by which this transfer might take place. The first is based on the fact that there is little restriction from a spatial point of view to the movement of electrons through a crystal lattice. Normally, in nonconducting inorganic crystals the most loosely bound electrons are attached to the negative ions. They cannot move about very much at ordinary temperatures, because more energy than is usually available at this temperature is required to move them from their position. At higher temperatures, of course, most crystals become conducting. When a quantum of light is absorbed by a bromide ion in silver bromide, the electron may be torn from its position and sent to another part of the crystal. There it settles on a silver ion, producing a silver atom. Very little energy is now required to move it from one silver to another, and in an electric field such a movement may readily take place, giving rise to the phenomenon of photoconductivity.

When the electron is removed from the bromide ion, it leaves it as a bromine atom. An electron from a neighbouring bromide ion can readily take the place of the original one, leaving in turn a bromine atom. In other words, the position of the bromine atom can move about through the crystal lattice. If it by chance comes to the surface it can, in the case of the photographic plate, readily oxidize an adsorbed gelatine molecule. The silver atom is then stabilized and its position moves about until it meets another silver atom, preferably at a surface, where it starts the nucleus of a crystal. This forms the latent image of the photographic plate which, on development, is increased about ten millionfold. Crystals not already having nuclei of silver do not develop in the period of time in which the plate is immersed in a reducing solution. The whole question of the action of a photographic plate is much more com. plicated than this, but the above mechanism provides a good working hypothesis.

The second important mechanism for the transfer of energy through a crystal does not require even the movement of an electron. According to this, the primary process of light absorption results (as usual) in an electron with a higher amount of vibrational energy, but moving more or less in its original mean position. Such a vibrating electron produces an oscillating electric field which influences neighbouring electrons, and the energy of the electron, the 'exciton', is passed on from one electron to another as a complete unit. In this way the exciton 'diffuses' about until it reaches a surface where it may cause a chemical change, or until it reaches a potential radiator where it may result in fluorescence.

There are many examples similar to the photographic plate in which light absorbed in the interior of a crystal leads to a reaction on the surface. Most inorganic oxides, such as zinc, titanium and antimony, which absorb in the near ultra-violet, can cause a photochemical change with certain organic compounds adsorbed on their surface. In no case as yet has it been established whether the transfer occurs by free electron diffusion or by exciton diffusion.

\section{Obituary Notices}

Prof. S. L. Loney

GIDNEY LUXTON LONEY, whose death at the $\checkmark$ age of seventy-nine years occurred on May 16, belonged to the older generation of Cambridge mathematicians, whose training was broad as well as specialized, and his position as third Wrangler in the then undivided Tripos justified the high opinion formed of his abilities at Tonbridge School by his teacher, Mr. Hilary, one of the most remarkable mathematical teachers of his generation.

On leaving Sidney Sussex College, Cambridge, Loney was faced with the choice which so often confronts the ablest young graduates - the choice between the advancement of specialized learning by research, or the advancement and diffusion of sound knowledge by direct teaching. His exceptional gifts of clear exposition and fertility of illustration led him towards teaching, and he devoted himself wholeheartedly to his chosen profession, as professor of mathematics at Royal Holloway College, London. Before the establishment of the internal side of the University of London, many students of Holloway College took the Final Honour Schools of the University of Oxford, and the mathematical class lists of the years 1891-1907 afford evidence of the high standard of mathematics at the College at that time. After the College became one of the internal colleges of the University of London, a correspondingly high standard was maintained in the London honours lists, and this tradition has been continued under his successors, Prof. Jolliffe and Prof. Bevan Baker.

It was during his tenure of the College professorship of mathematics that Loney's text-books on applied and pure mathematics were written; and for nearly fifty years they have been established as 